\title{
Zur Constitution des Chinins und Chinidins.
}

\author{
Von Zd. H. Skraup.
}

(Aus dem Laboratorium der Wiener Handelsakademie V.)

(Vorgelegt in einem versiegelten Couvert am 20 Juli 1882.)

Vor einem Jahre habe ich eine Untersuchung „Über Chinin und Chinidin" veröffentlicht (Sitzungsber. LXXXIV. Bd. II. Abth. 622), deren wichtigste Resultate sich folgendermassen zusammenfassen lassen:

„Beide Alkaloide gehen mit Chromsäure oxydirt in Kohlensäure, eine syrupöse und in eine gut krystallisirte Säure uber, welch letztere Chininsäure genannt wurde.

Die Chininsäure hat die Zusammensetzung $\mathrm{C}_{11} \mathrm{H}_{9} \mathrm{NO}_{3}$, ist einbasisch und einatomig; mit concentrirter Salzsäure erhitzt, liefert sie Chlormethyl und Xanthochinsäure der Formel $\mathrm{C}_{10} \mathrm{H}_{7} \mathrm{NO}_{3}$.

Die Xanthochinsäure wird endlich beim starken Erhitzen in Kohlensäure und in einen phenolartigen Körper zerlegt, der seiner Entstehung halber als ein Oxychinolin anzusehen wäre.

Nachdem nun aber, wie ich in derselben Mittheilung nachwies, die Chininsäure mit Kaliumpermanganat oxydirt, dieselbe Tricarbopyridinsäure liefert wie die Cinchoninsäure, ist es erstlich mehr als wahrscheinlich, dass sie ein Chinolinderivat ist, zweitens ist es dann aber auch sicher, dass ihre Methoxylgruppe im Benzolring des Chinolins sitzt. Als Consequenz dessen ergibt sich die Annahme, dass auch das aus der Xantochinsäure entstehende Oxychinolin ein im Benzolrest hydroxylirtes Chinolin ist."

Letzterer Körper ist damals nur sehr beiläufig charakterisirt worden, da Rücksichten anf die immerhin umständliche Beschaffung des so kostbaren Materials es räthlich erscheinen liessen, die Untersuchung erst dann vorzunehmen, wenn die synthetisch darstellbaren Oxychinoline gentigend gekannt sind.

Nach unseren gegenwärtigen Vorstellungen sind nur vier im Benzolrest hydroxylirte Chinoline zu erwarten, es dürfte dann 
also nicht schwer fallen, das "Chinin-Oxychinolin" mit einem der drei bereits bekannten Isomeren zu identificiren, oder es als das vierte Oxychinolin zu erkennen, dessen synthetische Darstellung bisher nicht gelungen ist.

Es sei vorausgeschickt, dass der Vergleich die Natur des Oxychinolins aus Chinin klarstellte; nachdem die Thatsache nicht ohne Wichtigkeit ist, erscheint es mir nothwendig, die dahin führenden Versuche detaillirt zu beschreiben.

Die Xantochinsäure wird erst bei hoher Temperatur zerlegt, bei $310^{\circ}$ ist sie noch vollständig unzersetzt. Zur Darstellung des Oxychinolins wurden daher $2 \mathrm{Grm}$. reine Säure in einem mit Gasableitungsrohr versehenen Kölbchen im Eisenfeilbade bis zur beginnenden Gasentwicklung erhitzt, die Temperatur dann so regulirt, dass der Gasstrom gleichmässig blieb.

Die Xanthochinsäure schmolz zu einer schwärzlichen Flüssigkeit, in den oberen Theile des Kolbens sublimirten blendend weisse Prismen, denen eine sehr kleine Menge einer gelblichen Substanz beigemischt war, offenbar der Zersetzung entgangene Xanthochinsäure. Das entweichende Gas tribte in allen Stadien des Processes Barytwasser sehr stark.

Nach Beendigung der Gasentwicklung wog der erkaltete Kolbeninhalt 1.51 Grm., $2 \mathrm{Grm}$. Xanthochinsäure hatten demnach 24.5 Percent an Gewicht verloren, während die Gleichung.

$$
\underbrace{\mathrm{C}_{10} \mathrm{H}_{7} \mathrm{NO}_{3}}_{\text {Xanthochinsäure }}=\underbrace{\mathrm{C}_{9} \mathrm{H}_{7} \mathrm{NO}_{3}+\mathrm{CO}_{2}}_{\text {Oxychinolin }}
$$

einen Verlust von $23 \cdot 2$ Percent erfordert.

Die sublimirten Krystalle erwiesen sich als leicht löslich in verdünnter Kalilauge und Salzsäure, nicht löslich in kaltem, sehr schwer löslich in heissem Wasser, aus der salzsauren Lösung wurden sie durch Sodalösung wieder gefällt, besassen also die allgemeinen Eigensehaften der Oxychinoline. Nachdem nun aber die Substanz weder mit Wasserdampf flüchtig war, noch in verdünntem Alkohol gelöst und mit Eisenchlorid versetzt eine Grünfärbung, sondern eine röthliche zeigte, konnte sie mit dem synthetischen 0 -Oxychinolin nicht identisch sein.

Behufs weiterer Untersuchung vereinigte ich das Sublimat und den am Boden des Kolbens befindlichen krystallinischen 
Kuchen, löste in verdünnter Salzsäure und liess bei gewöhnlicher Temperatur eindunsten.

Das wieder nun in Wasser aufgenommene Chlorhydrat, von harzigen Flocken abfiltrirt, war dunkelbraun gefärbt, es verlor den grössten Theil der färbenden Substanzen, indem es kochend mit einigen Tropfen Platinchlorid vermischt, die helle Lösung filtrirt und dureh wiederholtes Einleiten von Schwefelwasserstoff das Platin ausgefällt wurde. Beim Eindampfen hinterblieben dann gelbe Prismen. Vorerstaus kochendem Alkohol krystallisirt, wurden sie dann in Wasser gelöst; durch Zusatz von etwas Zinnchlorïr, Ausfällen mit Schwefelwasserstoff, entstand eine nahezu farblose Flüssigkeit, die sehr stark concentrirt und dann mit etwas Alkohol versetzt etwa $1 \mathrm{Grm}$. eines schwach gelblich gefärbten Salzes in hübschen Prismen lieferte.

Dasselbe gab nun in Wasser gelöst und mit Ätzkali, schliesslich mit Natriumearbonat ausgefullt, schwach gelbliche Krystallkörner, die gewaschen und aus absolutem Alkohol umkrystallisirt, in fast weissen Prismen anschossen.

Ein Theil derselben wurde zur Darstellung der Platindoppelverbindung benittzt, die röthlich gelbe Prismen, sehr schwer in kaltem, leichter in heissem Wasser löslich, bildet.

$0.2606 \mathrm{Grm}$. verloren bei $100^{\circ}$ getrocknet $0.0133 \mathrm{Grm} . \mathrm{H}_{2} \mathrm{O}$ und hinterliessen 0.0682 Grm. Pt.

$$
\frac{\begin{array}{c}
\text { Berechnet für } \\
\left(\mathrm{C}_{9} \mathrm{H}_{2} \mathrm{NO}\right)_{2} \mathrm{H}_{2} \mathrm{Cl}_{6} \mathrm{Pt}+2 \mathrm{H}_{2} \mathrm{O}
\end{array}}{\mathrm{Pt} \ldots 26 \cdot 49} \quad \underbrace{5 \cdot 10}_{\begin{array}{r}
26 \cdot 17 \\
\mathrm{H}_{2} \mathrm{O} \ldots 4 \cdot 89
\end{array}}
$$

Diese Zahlen bestätigen die erwartete Zusammensetzung.

Das Oxyehinolin aus Chinin krystallisirt aus absolutem Alkohol in ziemlich derben, aus verdünntem Alkohol in zarten, feinen Prismen, Alkohol löst es ohne Färbung, auf Wasserzusatz zeigt sich lichtgelbe Farbe, die nach Zufügung von Alkohol wieder verschwindet; keine Lösung zeigt irgend welche Fluoreszenzerscheinung, es kann also auch das -m-Oxychinolin, das insbesondere in verduinnt alkoholischer Lösung prächtig grün fluoreszirt, nicht vorliegen.

Der Schmelzpunkt ziemlich compacter Krystalle liegt aber bei $194^{\circ}$ uncorr., also sehr nahe dem des -p-Oxychinolins, der 
mit $193-194^{\circ}$ ermittelt worden ist. Folgende Angaben setzen ausser allen Zweifel, dass thatsächlich letzteres aus der Xantochinsäure entsteht.

Die alkoholische Lösung wird von Eisenchlorid um so schwächer und um so ausgesprochener gelb gefärbt, je reiner die gelöste Substanz ist.

Sie liefert nach Zufügung von Pikrinsäure alsbald zarte, gelbe Prismen, deren Schmelzpunkt glatt bei $235-235.5$ uncorr. liegt.

Nach Zusatz von Kupferacetat entsteht unmittelbar eine schön blaugrüne Färbung, dann allmählig gut ausgebildete violet gefärbte Prismen, die von kochendem Alkohol mit schön laubgrüner Farbe aufgenommen werden, nach dem Verdunsten unverändert hinterbleiben. ${ }^{1}$

Das Oxychinolin wird von etwa der vierfachen Menge concentrirter Salpetersäure in der Kälte nicht gelöst, leicht bei vorsichtigem Erwärmen. Die rasch mit Wasser wieder abgekuhlte Flüssigkeit setzt nach vorsichtigem Verdünen mit kaltem Wasser orangerothe Krystalle ab, die von kaltem Wasser schwer, von heissem leicht gelöst werden. Die Lösung vorsichtig mit Ätzkali versetzt, lässt zarte, gelbliche Prismen fallen, die aus verdiunntem Alkohol umkrystallisirt bei $140-141^{\circ}$ schmelzen, also bei derselben auffallend niedrigen Temperatur, wie das Nitroparaoxychinolin.

Ihre alkoholische Lösung wird nach Zusatz von Kupferacetat zuerst prächtig grun, und setzt dann einen kupferbraunen Niederschlag $a b$, wird aber gleichzeitig eine Spur Alkali zugefugt, einen grïnen. Das Barytsalz der Nitroverbindung bildet orangerothe seidenglänzende Nadeln und ist schwer in kaltem weit leichter in kochendem Wasser löslich.

Es sei ausdruicklich hervorgehoben, dass obige Reactionen gleichzeitig mit dem synthetisch erhaltenen Oxychinolin ausgeführt wurden und sich dabei vollständigste Übereinstimmung ergab.

1 Die Beschreibung der Kupferverbindung des synthetischen $-p-$ Oxychinolins weicht, was die Färbungen betrifft, etwas ab. Sublimirte synthetische Substanz verhält sich aber genau so, wie oben beschrieben. 
Dass die Schmelzpunkte nicht absolut übereinstimmen, kann bei so schwierig rein darstellbarer Substanz kaum Wunder nehmen, die Abweichung bei derselben ist uiberdies so gering, die sonstige Übereinstimmung so präcis, dass die Identität beider Körper ausser allem Zweifel steht.

Durch diese Thatsache wird die Constitution des von Königs als Chinolidin bezeichneten sogenannten Oxylepidin, wie es Butlerow und Wischnegradsky zuerst erhielten, aufgeklärt, es ist der Methyläther des - $p$.Oxychinolins oder des - $p$-Chinanisol, ebenso ist die Constitution der Xanthochin- und der Chininsäure bis auf die Stellung der Carboxylgruppe erkannt ${ }^{1}$ and damit die eine Hälfte vom Molekiul des Chinins und Chinidins.

1 (Zusatz, beigefügt am 19. Juli 1883.) Die Untersuchung ïber das $\alpha$ - und $\beta$-Naphtochinolin von A. Cobenzl und mir (Monatshefte IV, 436) hat gezeigt, dass die Stellung der Pyrichinmonoearbonsäuren und einiger Chinolincarbonsäuren thatsächlich so ist, wie ich es vor längerer Zeit (Monatshefte I, 800) vermuthungsweise ausgesprochen habe.

Damit ist nun auch die Stellung der Carboxylgruppen in der Chinchoninsäure, Chininsäure und Xanthochinsäure gegeben, die Constitution der Süuren durch folgende Formeln ausdrückbar :
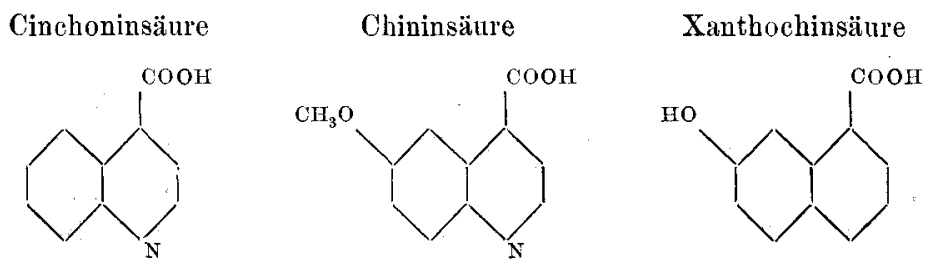